Cinémas

Revue d'études cinématographiques

Journal of Film Studies

\title{
Annonciations (Migrations, 3 )
}

\section{Jacques Aumont}

Volume 12, numéro 3, printemps 2002

Cinélekta 4

URI : https://id.erudit.org/iderudit/000735ar

DOI : https://doi.org/10.7202/000735ar

Aller au sommaire du numéro

\section{Éditeur(s)}

Cinémas

\section{ISSN}

1181-6945 (imprimé)

1705-6500 (numérique)

Découvrir la revue

\section{Citer cet article}

Aumont, J. (2002). Annonciations (Migrations, 3 ). Cinémas, 12(3), 53-71. https://doi.org/10.7202/000735ar

\section{Résumé de l'article}

La scène canonique de l'Annonciation a été traitée par la peinture, durant deux ou trois siècles, comme un défi à la mise en scène : comment conjoindre le céleste et le terrestre ? Des solutions trouvées pour ce " dispositif annonciatif ", cet article cherche à interroger la reprise dans certains films. On s'intéresse surtout aux films qui, sans citer littéralement aucun tableau, manifestent une " migration » de la peinture, par leur souci de produire du figuratif en réinterrogeant à neuf la situation symbolique, sans se reposer sur des êtres de fiction plus ou moins fantastiques. Ainsi, « faire des Annonciations ", pour certains cinéastes, n’a pas été une volonté d'ennoblir culturellement leurs films, mais la démonstration d'un désir de manier ensemble des formes, des thèmes et des figures - c'est-à-dire de produire, non un geste de peintre, mais un geste d' auteur.
Ce document est protégé par la loi sur le droit d'auteur. L'utilisation des services d’Érudit (y compris la reproduction) est assujettie à sa politique d'utilisation que vous pouvez consulter en ligne.

https://apropos.erudit.org/fr/usagers/politique-dutilisation/ 


\title{
Annonciations \\ (M igrations, $3^{1}$ )
}

\section{Jacques Aumont}

\begin{abstract}
RÉSUMÉ
La scène canonique de l'Annonciation a été traitée par la peinture, durant deux ou trois siècles, comme un défi à la mise en scène: comment conjoindre le céleste et le terrestre? D es solutions trouvées pour ce «dispositif annonciatif », cet article cherche à interroger la reprise dans certains films. On s'intéresse surtout aux films qui, sans citer littéralement aucun tableau, manifestent une «migration » de la peinture, par leur souci de produire du figuratif en réinterrogeant à neuf la situation symbolique, sans se reposer sur des êtres de fiction plus ou moins fantastiques. Ainsi, «faire des Annonciations», pour certains cinéastes, n'a pas été une volonté d'ennoblir culturellement leurs films, mais la démonstration d'un désir de manier ensemble des formes, des thèmes et des figures - c'est-à-dire de produire, non un geste de peintre, mais un geste d'auteur.
\end{abstract}

\section{ABST RACT}

In painting, the canonical scene of the Annunciation was, for a period of two or three hundred years, a challenge to mise en scène: how to join the heavenly and the earthly? This article inquires into how certain films have taken up some of the solutions that were found for this "annunciative model." The particular focus is on films which, without quoting any particular painting literally, show signs of a "migration" of painting into the cinema through their concern for creating a representational art by reexamining from scratch the question of symbolism, without relying on more-or-less fantastic fictional beings. Thus for certain filmmakers, 
"creating Annunciations" has not meant a desire to ennoble their films culturally, but is rather the manifestation of an attempt to wield forms, themes and figures as an ensemble. In other words, to produce, not the gesture of a painter, but the gesture of an auteur.

\section{$M$ ises en scène toutes faites ou à refaire (les Annonciations)}

Je reviens rapidement sur des questions déjà abordées, un peu différemment, dans des textes antérieurs, autour de la possibilité - culturelle, formelle, matérielle, idéologique et essentielle de repérer, dans des films et en général dans le cinéma, des élé ments picturaux. Cinéma et peinture: le thème a fait florès voici une petite quinzaine d'années, nourrissant à la fin des années quatre-vingt - quand toute la critique remâchait à qui mieux mieux l'idée de la «mort du cinéma» - colloques, ouvrages², articles, dans l'espoir sans doute de trouver une issue positive à ce qui passait pour une crise. D epuis, I'hégélianisme prêt-àporter a un peu disparu, et la critique est passée à autre chose. Pourtant, la question demeure: visible pour visible, visuel pour visuel, «le» cinéma, ou du moins, sa part un peu plus savante et cultivée, n'a-t-il vraiment rien repris de «la» peinture, c'està-dire de l'histoire des arts figuratifs en 0 ccident de la Renaissance à l'abstraction?

Autour de cette question, j'ai cherché, sans l'approfondir suffisamment, à suggérer un rapport possible 3 : on retrouve, dans certains films, des motifs picturaux avérés et traditionnels, souvent lourds de signification, selon la voie du «symbolisme déguisé» qu'a si bien mise en lumière le travail de Panofsky. $O$ bjets de peinture, comme ces tables garnies qui durant deux ou trois siècles ont été attachées de manière univoque au thème fondamentalement chrétien de la Vanité - et avec ces objets, ce qui voyage, c'est leurs symbolismes. Si je repère, dans L'Annonce faite à $M$ arie, un jeu de scène très discret au cours duquel le héros pèle un citron en spirale, et le dépose sur une table chargée de nourriture, j'aurai le droit d'évoquer les citrons pelés, symboles du temps qui passe, dans la peinture flamande et hollandaise du XVII ; je prends alors tout en bloc, le motif figuratif et sa signification conventionnelle (car un citron, par lui-même, n'a aucune raison d'évoquer le temps). C'est un exemple, à mes 
yeux, de «migration » thématique et «motivique» (pas exactement la migration formelle postulée par Warburg).

Je me demande à présent si des migrations analogues peuvent affecter la mise en scène, et non plus seulement les motifs du cinéma représentatif et scénique. L'hypothèse est que la mise en scène pourrait aussi voyager en bloc, appuyée sur des situations, dramatiques ou picturales. Et la question serait de savoir si, en pareil cas, le cinéma importerait aussi des significations toutes faites, ou si, au contraire, il aurait plutôt tendance, en reprenant de la peinture un ensemble déjà fictionnel, à suivre une pente critique et «interprétative».

Ce qui est sûr pour commencer, c'est que le cinéma peut emprunter sa mise en scène à la peinture. La chute de Babylone ou la Saint-Barthélemy, dans Intolerance, sont une succession de tableaux, au sens peinture comme au sens thêatre. À cette mise en scènelà, si raffinée ait-elle été, le cinéphile n'a eu de cesse d'échapper. Le goût en cinéma aura toujours été cela: échapper au théâtre, au pompier; ne pas ressembler à de la mise en scène. II y a deux ou trois ans, un symptôme aigu de cette prescription fut Rosetta, des frères $D$ ardenne: une espèce de M ouchette qui aurait contaminé de son hystérie la caméra, et qui arpente une banlieue belge de la cour au jardin; la critique s'est alors clivée, entre une exaltation de cet œil obscène collé au cul de la pauvre fille, avec ses collants râpés - retour éternel du pompier sous les oripeaux naturalistes - et une détestation de ce trop de cinéma sur trop de théâtre. $0 \mathrm{n}$ aura compris que pour ma part, je crois que Bazin avait raison: le théâtre au cinéma n'est supportable qu'avoué, affiché comme thêâtre. (Et la peinture, idem ?)

Par ailleurs tout théâtre chez nous est chrétien. Le christianisme comme source de nos symbolismes et de leur mode d'emploi; comme conteur d'histoires merveilleuses: rencontres, surprises, métamorphoses; comme inventeur d'un thêâtre et d'un théâtral, intimement liés à «la» peinture (la nôtre), à ses effets lumineux, à son miracle intrinsèque (celui de la touche). Apparitions et épiphanies - le thème du voile des apparences, déchiré par le Transcendant pour que l'homme aperçoive un peu de vrai - mais transposées, du registre verbal, dans le registre dramatique, c'est-à-dire en fin de compte toujours celui du coup 
de thêâtre. Jésus pleurant, caressant, buvant et mangeant, Jésus lavé, fouetté, enterré: le christianisme n'est qu'événements humains et dramatisés. Tout ce qui se passe en présence des apôtres est théâtre, il y a toujours un dialogue pour susciter de la mise en scène, même pour les épisodes fantastiques (les langues de feu); par contagion, tout ce qui précède la vie publique de Jésus a été - au besoin, de toutes pièces - imaginé comme théâtre par la peinture, puis par le cinéma. Les premières vues Lumière dramatisées: des Passions. Parmi les premières grandes productions américaines: From the M anger to the Cross (De la Crècheà la Croix).

Pourtant la vie de Jésus n'est pas ce qu'il y a de plus propice à mise en scène (trop purement dialogique? ou au contraire, trop tendue vers la révélation?) ; c'est dans d'autres épisodes que la peinture a trouvé des réservoirs de théâtre, au premier chef, l'Annonciation. Si elle existe comme sujet de peinture, c'est à proportion de sa simplicité dramatique autant que de sa portée théologique. Situation banale, Boy M eets Girl. Un garçon ou ce qui a l'air d'un garçon, une fille ou ce qui n'est pas seulement une fille; une parole, entre eux, lourde de conséquences. Toute la scène consiste dans l'émission de ce message inattendu, et dans sa réception; il y a donc bien davantage que la scène, que le thêâtre; pourtant il n'y aurait rien s'il n'y avait pas cette scène. Cette rencontre n'est pas la simple coprésence de deux personnages, qui se résoudrait dans l'action; elle est la mise en contact miraculeuse de deux natures de l'être, le premier moment de I'Incarnation; elle dit l'essence même de ce dogme, la possibilité d'une nature double, à la fois terrestre et céleste. $M$ ais pour le dire, la rencontre reste indispensable - deux êtres, deux figures, deux personnages, une scène jouée.

$D$ ans les Annonciations peintes, le problème est bien un problème de scénographe et de metteur en scène: imaginer une solution pour figurer dans un même espace la rencontre d'une créature céleste, qui parle pour annoncer un futur et un accomplissement, et d'une créature terrestre, qui se tait ou répond humblement, et réagit par rapport au présent. C réature immaté rielle, l'Ange? pas de corps? pas de sexe? de quoi sont faites les ailes? le halo, l'auréole? Les anges de l'art chrétien sont des ava- 
tars et des migrations, venus des erôtes grecs - esprits ailés, messagers des dieux - et des genii romains - gardiens de l'âme et ses guides post mortem - , jusqu'à l'invention définitive des adolescents asexués de la Renaissance. Les décisions figuratives à leur sujet sont variables, mais ils sont toujours au bord du métamorphique, parfois confondus avec une nuée ou une lumière, qui doit trouver forme terrestre pour communiquer avec la Vierge. Celle-ci, au contraire, est une jeune fille simple, ordinaire; souvent, on sent que le modèle a été pris dans le peuple, on imagine des paysannes endimanchées, des filles d'artisans (Pasolini et Godard s'en souviendront).

Autre question, celle de la frontière entre le monde d'ici-bas, où une jeune femme lit un livre sous un auvent, près d'une fontaine, devant la porte d'une chambre où l'attend le lit, et le monde de là-haut, où sans que le temps existe les anges adorent Dieu perpétuellement. Les artifices pour faire sentir cette barrière sont affaire d'époque. Au XIV e ou XV siècle, les deux personnages sont séparés par une colonne, à moins que ce soit une trouée perspective; I'action se situe au croisement d'une frontalité et d'une profondeur; le dialogue est matériellement inscrit, par des lettres peintes à la peinture dorée, ou alors, traduit dans des gestes et attitudes codés (les cinq moments du «colloque angélique» et de la réaction de $M$ arie ${ }^{4}$ ). Les peintres rivalisent d'astuce pour matérialiser la double nature de la rencontre: colonne dorée qui se détache du fond tout en lui appartenant, plaques de marbre veinées qui exténuent la perspective en l'accomplissant, porte de hortus conclusus trop grande ou trop petite, etc. Au siècle suivant, c'est le merveilleux, la lumière, les nuées, les stucs qui unifient tout; on est passé à une scénographie du fantastique, contemporaine ou juste préfiguratrice des grandes machineries de théâtre. (Une autre façon de décrire serait de se demander à quel moment l'ange touche le sol, à quel moment il plane ou lévite.)

II y a des tableaux davantage surchargés de symboles (L'Adoration des $M$ ages de Bosch, par exemple); il y a des scènes de la vie du Christ plus propices à dramatisation (la mort, l'enterrement, la transfiguration). Aucun de ces sujets n'offre une conjonction aussi pleine, un équilibre aussi réussi : autant de drame patent 
que de symbolisme caché. L'Annonciation, en peinture, est un dispositif. Au reste, je cite littéralement un certain travail consensuel des historiens d'art, que le livre récent de $D$ aniel Arasse (1999) expose et prolonge avec maestria, auquel d'ailleurs j'emprunte mon savoir d'amateur. II reste que la peinture d'Annonciation a suscité, justement, cette quantité et cette qualité d'interprétation, plus qu'aucun autre épisode, Crucifixion incluse. La C roix est le symbole majeur, mais en dehors de symbolismes tout faits, comme le crâne-Golgotha, la mise en scène de la crucifixion est sans surprise; il n'a pas été jugé utile de la présenter au milieu de pièges optiques, de roueries de la repré sentation, de spéculations perspectives cachées. C'est que l'Annonciation est un drame parlant, avec dialogue - quand les Sept Paroles du Christ sont un monologue.

Comment cette machinerie, ce «dispositif annonciatif», peut-il se reprendre dans le cinéma de fiction - à supposer qu'il y ait un désir pour fonder cette reprise? D'abord la surface, les apparences: I'orientation latérale de la scène, les postures, l'architecture, les attributs plus ou moins littéraux des personnages, jusqu'aux plumes de l'ange si on y tient. Le seul ange de cinéma vraiment ailé qui me vienne à la mémoire est l'Archange du prologue de Faust (Murnau, 1926) ; ses plumes, plutôt ridicules, sont prises dans une pâte lumineuse qui en transfigure la matière visuelle; Murnau, connaisseur en peinture (selon le topo biographique), a visiblement cherché à la copier, mais pas à la lettre. En outre, cet ange est confronté à un autre ange, déchu celui-là, et cela ne fait pas une Annonciation. En cinéma comme en peinture, le cœur de la scène en effet, c'est ce point sensible: les deux créatures ontologiquement disparates, et la frontière visible entre leurs domaines respectifs.

II y a chez Pasolini, amoureux obsessionnel de la religion chrétienne, une fréquence inhabituelle de l'allusion à cette scène et à son dispositif. D ans Accattone, comme l'a observé Alain Bergala ${ }^{5}$, la rencontre du mendigot (c'est le sens du titre italien) et de la jeune Stella est une sorte d'Annonciation à l'envers, où l'annonciateur est une femme, et l'annunziato un garçon. Pasolini a pris un malin plaisir à parsemer la scène de références plus ou moins ostensibles, en insistant sur la différence des postures 
et des mouvements - elle debout, agitée, entourée d'un halo de fumée lumineuse, lui assis, attentif et grave sous sa désinvolture; en faisant expliciter par le garçon l'allusion contenue dans le prénom de la fille - «Stella, Stella, indicami'l cammino!»-, prophétesse ou messagère malgré elle; en les situant, bien écartés, de part et d'autre d'une espèce de cour fermée par une barrière de bois, «jardin clos» dérisoire, et en les séparant non par une colonne mystique mais par un tas de déchets, selon le thème pasolinien de l'ordure poétisée. Pourtant, cette accumulation de références expresses à la scène canonique échoue à rendre l'essentiel du moment annonciatif. II y a la scène, il y a le théâtre, mais il n'y a pas la faille mystique, la rencontre impossible et pourtant possible, la frontière symbolique et mystérieuse, la transformation profonde et l'annonce d'un autre monde. Après sa rencontre avec Stella, Vittorio va changer - mais dans les limites d'une destinée humaine; rien d'équivalent, même par métaphore, au destin de $M$ arie.

Pasolini a dû sentir cette butée, alors même que l'idée l'attirait par sa condensation de symbole et de théâtre. Son œuvre de cinéma questionne sans cesse la forme du colloque angélique, autour d'une solution unique, celle du faceà-face. L'ange énigmatique de Théorème, dont l'évangile est confus (quelle bonne nouvelle apportet-il ?), est destiné à changer le sort de cinq personnes - bourgeois et employés, hommes et femmes, adolescents et adultes, tous ordinairement humains, quand lui vient d'ailleurs. Cet ange qui caresse et possède, on ne nous le montre jamais faisant qu'une seule chose: fixer du regard, sans intention, sans désir, comme le serpent hypnotise la proie. La forme proposée par le cinéaste est aussi sans intention; elle évacue toute identification à un regard; c'est un raccord entre des regards renvoyés frontalement, longuement, sans ciller; un raccord qui lie et délie à la fois, insiste discrètement sur la faille ontologique, la déhiscence entre les regardeurs.

Au tout début du Vangelo, lorsque Joseph regarde, morose et fâché, $M$ arie enceinte, c'est aussi un affrontement direct des yeux qui dit la fêlure entre eux: une fêlure justiciable de la psychologie et des mœurs, mais que le raccord brutal accentue, laissant percer le soupçon qu'elle excède les justifications humaines. 
Q uand, peu après, Joseph voit I'ange en songe, la forme est modifiée par le tremblement de la caméra portée à l'épaule, qui suggère l'hésitation de Joseph (doit-il croire ses yeux? ses oreilles?). Ainsi, c'est paradoxalement dans le face-à-face de I'homme et de l'ange que le raccord est psychologisé; au retour chez $\mathrm{M}$ arie, même si les mimiques, de tristes, sont devenues joyeuses, la séparation des deux personnages par les plans reste aussi rigide. Le choix figuratif est on ne peut plus clair : l'ange peut-être n'était qu'un rêve, le produit fantomatique d'un esprit humain; $M$ arie, elle, est bien ailleurs - dans un monde auquel Joseph n'accédera jamais.

Avec la séduction de Laura Betti par l'immobile et obscène Terence Stamp dans Théorème, et plus encore, avec la scène de consultation de la Pythie dans $\mathbb{E}$ dipe roi, toute psychologie disparaît. $\mathbb{E}$ dipe est confronté longuement à son D estin : d'abord fondu dans la foule des suppliants, puis peu à peu amené vers le face-à-face, seul. Sergio Citti épuise toute la gamme des mimiques; il est tout à tour tranquille, content, égaré, épouvanté: toujours de face, toujours regardant vers nous, toujours monté en alternance avec la Pythie, masquée, vulgaire, qui mange, s'accroupit comme pour chier ou accoucher, vaticine, rit. Face à la devineresse, il est un mortel en proie à l'angoisse; mais le champ-contrechamp à $180^{\circ}$ - forme interdite, sulfureuse, un diabolus in cinematographo comme il existe un diabolus in musica - empêche toute résolution, toute thaumaturgie; la femme doit rester à distance infinie, démente comme son rire; enceinte, elle est porteuse d'un avenir interdit au malheureux qui croyait se libérer. La scène est surchargée de symboles déguisés, par exemple l'arbre sous lequel la pythonisse est installée, un jujubier: Zizyphus spina Christi, l'arbre dont les rameaux épineux formèrent la couronne du Christ - et aussi le symbole islamique (le film est tourné au $M$ aroc) de la mesure de l'espace et du temps, dont le fruit est nourriture d'immortalité.

Après la séance (Pasolini ne dissimule pas le parallèle avec une psychanalyse), $\mathbb{E}$ dipe s'en va en chavirant, ivre de la révélation qu'il vient de subir. Le contrechamp sur son regard montre seulement le désert, là où juste avant c'était une foule (là où sétait une foule, comme écrirait un critique lacanien). M ais ce n'est 
pas seulement un trait de psychologie pour montrer qu'il est égaré, perdu: c'est la conclusion nécessaire, figurale, de cette forme inventée par le cinéaste et qu'ici il mène à son terme. Champ contre champ: le film prend la préposition à la lettre; il faut que l'un des deux antagonistes cède, disparaisse ou se transforme. Annonciation d'un destin atroce par une messagère perverse: le monde des dieux a frappé la scène; le monde des hommes ne peut plus être montré optiquement; la forme l'a changé. Tout cela est la conséquence logique de la prise au sérieux du «contre», de la rigueur oppositive d'une forme cinématographique pourtant banale.

Avec cette scène de l'ivresse d' $\mathbb{E}$ dipe, de la révélation hallucinante qui ne le lâche pas et fait vaciller les apparences devant lui, Pasolini mène aussi loin que possible la logique du dispositif annonciatif. Tous les éléments sont là: le messager, ni homme ni dieu; le destinataire purement humain; le monde des dieux d'où provient le message; le choc des mots divins sur le pauvre mortel ; la blessure symbolique (dans l'Annonciation, cette blessure occasionnée par le rayon d'or du Saint-Esprit est occultée par le geste d'autoprotection des mains de M arie, rabattues devant sa poitrine) ; la transformation radicale et définitive de I'annunziato. Comme dans Accattone, la mise en scène et le découpage du film se soumettent à la forme picturale dans ses principes profonds (sauf qu'ici, le face-à-face remplace totalement la vue latérale). En même temps - et à la différence de l'Évangile - aucune œuvre de peinture n'est citée, n'est même évoquée. Migration? en un sens, oui, car il y a bien eu un voyage, inattendu, risqué, de ces principes picturaux, qui sont détournés, changés, immigrés. Seulement, ce ne sont pas des figures qui ont migré. C'est une migration intellectuelle, une migration de schèmes mentaux et symboliques.

Lorsqu'il adapte la légende mariale dans Je vous salue M arie, Godard s'écarte encore davantage d'un retour littéral de la peinture. La figure angélique est démultipliée, d'abord sous les espè ces de l'avion dont on ne sait s'il atterrit ou décolle et qui écho affaibli de la lune tranchée de Buñuel - traverse ou transperce, moustique presque invisible, un immense soleil orange. Ensuite, c'est ce couple tout de guingois, avec un adulte nommé 
Gabriel, comme chez $Q$ ueneau, et une petite fille qu'on a envie, du coup, de baptiser Zazie (elle en a l'effronterie). M ais l'Ange, ce n'est pas $\mathrm{G}$ abriel, c'est ce couplelà - à la fois homme et femme, adulte et enfant - , manière pour G odard de dire qu'un ange, surtout celui de l'Annonciation, n'est pas figurable simplement comme un humain. Le film insiste, pour les obtus ou les distraits: Gabriel et la petite nouent à deux un lacet de soulier (c'est si difficile que les acteurs n'y arrivent qu'à peine) : un seul lacet pour deux mains différentes, un seul être pour deux corps. Pas humain. D e même qu'ils ont, à la parole, un rapport dédoublé: citations, allusions, syntagmes figés (au risque de se tromper, de citer les mauvais énoncés, ceux qui n'ont rien à voir avec I'histoire, comme le fait Gabriel au début, dans le taxi - et la petite al ors le corrige).

$\mathrm{M}$ arie au contraire a bien des attitudes et des réactions de jeune fille, même si la mise en scène la tire sans cesse vers autre chose: une simplette, sœur de la Cléopâtre des $C$ arabi niers, lorsqu'elle revient à cloche-pied, ou lorsqu'elle empêche son père de cogner; une sainte ou une magicienne, dans sa proximité métonymique avec la Lune. La longue scène dans la station-service, toute distanciée qu'elle est ( «fais pas l'innocente», lance G abriel à $M$ arie), est bel et bien une Annonciation ; elle mêle des retours brusques et inattendus des conventions du Q uattrocento, et des digressions burlesques ou dérisoires. Joseph, mué en chauffeur de taxi, voit opposer un feu rouge à son désir pour $M$ arie après qu'elle a été sanctifiée par l'Annonce; il lit un texte prophétique à sa façon, $D$ emain les chiens; I'ange-fillette vole à $M$ arie sa parole (comme dans le panneau siennois de Simone M artini elle va matériellement, sous forme d'inscription, d'une bouche à une bouche) ; M yriem Roussel adopte successivement les expressions codées de la surprise (conturbatio), de la demande (interrogatio), de l'acceptation (humiliatio), comme dans le sermon de Fra Roberto ${ }^{6}$.

Comme Pasolini, Godard est d'accord pour assimiler un héritage. $M$ ais assimiler ne veut pas dire calquer ou dériver; cela veut dire refaire, et à neuf. D émontage et remontage conscient, désarticulant la scène en ses éléments premiers, personnages, lieux, accessoires, moments, mimiques, postures - pour la 
recomposer avec les mêmes éléments dans un autre ordre, et chacun, subtilement (la lune) ou grossièrement (le couple d'anges) changé. La conturbatio de la Vierge est signifiée quand $M$ arie entend passer l'avion et se fige, l'air troublé (sans raison, car les avions passent toujours à la même heure, elle ne devrait pas être étonnée) ; puis de nouveau, et cette fois sel on le code des attitudes, par un retrait de son corps et de son visage au milieu de la scène de la station-service (mais nullement au début de la scène, comme on l'aurait attendu). D e même, son humiliatio, sa meritatio, sont signifiées par des gestes ou des airs du visage qui peuvent être lus aussi selon l'ordre du réalisme. La peinture est noyée sous ce remontage, elle n'est plus un trésor (de représentations) qu'on pille, mais une fontaine (de figuration) qui coule.

$D$ ans l'Annonciation, il y a l'annonce et son messager, le message et sa destinataire. II y a tous les affects humains que peut déclencher la confrontation soudaine d'une jeune fille vierge à un garçon d'une beauté angélique, et qui lui annonce qu'elle va être l'instrument du salut. II y a une vie quotidienne et humble, celle du travail et de la modestie féminins, troublée par la vie glorieuse de l'Incarnation. Voilà pour le drame, et ramené à ses plus simples termes il est extrêmement banal : une rêverie ou un fantasme de jeune fille exaltée (au moment où s'invente le cinéma, on a tendance à penser que la virginité côtoie l'hysté rie); ou bien, trivialement, une scène de séduction. Séduction de l'invisible, fantasmatique ou fantastique: voilà bien un maté riau à cinéma, et de ces rencontres entre créatures comme vous ou moi et créatures "angéliques», les films sont prodigues, même au sein des genres réalistes.

$M$ ais fabriquer une rencontre ne suffit pas. Les créatures des Ailes du désir ont tout ce qu'il faut, l'air humain et l'air céleste, la mémoire et la capacité de surécoute, l'accès à l'infini et la mauvaise conscience. Pourtant il n'est guère de film qui soit aussi éloigné de tout sous-venir de la scène annonciative; les confrontations entre la jeune femme et l'ange multiplient les signes du surnaturel, mais selon une logique triviale, qui ne fait que transposer l'humain dans le registre du surhumain. L'ange peut entendre ce qui est «dans la tête», mais ce n'est qu'une tête 
pauvrement humaine, pleine de sentiments normaux, et l'entendre n'est que l'exacerbation d'une capacité humaine, c'est-à-dire une fois de plus la constatation attristée de «nos» limites. Du coup, la métamorphose finale de l'ange, son accès euphorique à la sensation - les couleurs avec leurs noms, le goût du sang sont un pur coup de force du récit, sans rapport avec le cinéma (un comble, dans une œuvre qui joue autant avec le signifiant filmique).

II n'y a pas de règle générale, en tout cas je n'en vois pas. $M$ ais ce qui fait que Pasolini ou Godard, chacun à sa manière, a vraiment touché à I'héritage, a vraiment produit une transmigration, c'est leur souci de produire du figuratif en réinterrogeant à neuf la situation symbolique, sans se reposer sur des êtres de fiction plus ou moins fantastiques. Le champ-contrechamp «absolu » de l'un, le démontage et les équivalences de l'autre, travaillent l'intimité du cinéma: le cadrage, le montage, les vitesses, le jeu du centre et des bords. Autrement dit, ils s'en prennent à ce qui, dans l'image de film, peut faire figure. La logique figurative de l'Annonciation, c'est la coprésence de deux entités ontologiquement hétérogènes, au prix d'un travail de couture paradoxale de l'espace visible. Cette logique n'implique pas qu'on se prononce sur les pouvoirs des êtres surnaturels mis en scène - mais seulement, qu'on respecte leur relation à l'humain avec lequel ils colloquent, et qu'on sache mettre en évidence le paradoxe spatial.

Simon le stylite, dans le film de Buñuel, est confronté à un ange malin - un diable femelle et agaçant - par un jeu ironique sur l'espace, qui tient les deux figures à distance et à proximité à la fois (c'est de l'ironie que provient le paradoxe). D ans ces scènes de tentation extrême, la colonne sur laquelle se tient l'ermite peut jouer comme écho décalé, un peu incongru, de la colonne des Annonciations peintes; elle symbolise et figure la mise à distance des affaires humaines, donc une garantie de sécurité spirituelle, près du ciel; en même temps, le saint y est isolé, donc à la merci de qui viendra le rejoindre, comme c'est le cas, malicieusement, de la démone, laquelle se retrouve aussitôt à proximité7. Colonne qui sépare les espaces et les réunit; espace paradoxal, proche et lointain à la fois; créature «angéli- 
que» (un mauvais ange, un messager du $M$ alin). II est vrai que Simon del desierto est un film imprégné de christianisme, comme le seraient, analysables de la même manière, Bad Lieutenant et son Christ décrucifié, ou le H érode du début de Salomé de Bene, avec sa vision érotique d'un ange trouble, ou la première scène dans la bibliothèque du couvent d'O liveira, avec ses raccords abrupts sur un homme pris en tenaille entre un diable et un ange ${ }^{8}$. Chez Ferrara, la figure observée ou hallucinée par le héros se métamorphose dans le raccord : statue de bois, figurant en chair et en os, personnage du drame; le «colloque» est dérisoire, peu angélique, voire blasphématoire; mais juste avant, c'est la conversation de $\mathrm{H}$ arvey Keitel avec la jeune nonne qui a été violée (et qui, elle, fait bien figure d'ange) : deux lumières à l'arrière-plan y dessinent deux espèces de chapeaux lumineux et coniques, répondant à la cornette de la religieuse; l'espace est ainsi unifié et dédoublé à la fois, mais en terrain purement figuratif. Etc.

Les exemples sont illimités, parce que ce à quoi un personnage de film peut être confronté en matière de surhumain est sans limite. Le héros du Garçon aux cheveux verts - une fable réaliste, au lendemain de la guerre, avec des leçons très terre-àterre sur les enfants victimes, sur la politique, sur la chasse aux sorcières, sur l'Amérique et la vie quotidienne - est un emblème dans une allégorie. O rphelin de guerre, il prend à son compte toutes les horreurs et toutes les peines; la scène clé est encore une confrontation, mais elle est dédoublée. II est d'abord mis en face d'affiches de propagande, qui représentent tous les possibles de la misère enfantine; plus tard, il retrouve, vivants, les personnages des affiches, au sein d'un décor de ruines, dans une clairière. La scène est indécidable (hallucination? métaphore pure?) mais elle fonctionne bien comme une apparition, et même, si l'on veut, comme une annonciation, puisque ces anges laïques expliquent au garçon la signification de ce qui lui est arrivé et qui est humainement incompréhensible (les cheveux verts). L'espace n'est pas dédoublé par la mise en scène, mais par le découpage, reliant l'espace de l'apparition (les enfants dans la clairière, leur discours) et l'espace tout différent des affiches (les enfants représentés, parlés par les légendes des photographies). 
Le garçon, qui reste le même d'une scène à l'autre, affirme son humanité - tandis que les apparitions deviennent fantastiques, des messagers d'on ne sait quelle transcendance (ou immanence). $D$ ans ce drame «politique» fait par une équipe «de gauche» (le producteur, les deux scénaristes, le réalisateur furent blacklistés), le schème annonciatif est d'autant plus voyant qu'il est inattendu.

Je reviens à un film chrétien - mais au symbolisme déguisé, souterrain. Scottie dans Vertigo voit M adeleine, puis Judy, comme des apparitions, de nature et d'allure différentes; son souci est de les réduire à une seule apparition. C'est l'enjeu des deux scènes de l'hôtel, dans le second volet ou le second pli du récit. La première fois, Scottie raccompagne Judy; il la drague, ou c'est ce qu'elle feint de croire pour le tenir à distance; de cette distance maintenue, il la voit, profil silhouetté devant la lumière verte, d'autant plus ressemblant au modèle absent qu'il est réduit à ce qui ne peut tromper (le contour). Seconde scène à I'hôtel, avec Judy en M adeleine, tailleur gris, cheveux blonds, le prétexte de la coiffure à corriger qui l'envoie dans la salle de bains, et nous permet de la voir - toujours par ses yeux à lui sortant au milieu d'une aura, verte aussi. D 'une scène à l'autre, le violet qui était d'abord sur la robe est passé sur les murs de la chambre, discret, léger, une ombre. Le baiser, lui, est un remake de celui de Notorious, aussi long, aussi malaisant (mais pour d'autres raisons: on sent le peu d'attirance physique de James Stewart pour Kim N ovak). La magie aussi en est plus extérieure: à l'intimité prolongée obtenue par le mouvement d'appareil virtuose des Enchaînés succède le truquage par lequel la chambre d'hôtel soudain devient la remise des voitures de la mission espagnole. La chambre tourne, à moins que ce ne soit la tête de Scottie, vertigo: I'apparition, finalement, avait bien un pouvoir surnaturel, elle étourdit, emporte, déroute.

Vertigo est un film double, chacun le sait, et cette (double) scène de séduction d'un vivant par une morte-vivante à son tour s'éclaire d'une autre scène d'apparition, plus énigmatique dans sa simplicité, au cœur du premier pli de l'œuvre. D éguisée en $M$ adeleine, Judy désigne sur la coupe du tronc de séquoia la date de sa naissance et de sa mort, des dates du siècle précédent. 
Elle s'éloigne, et soudain elle n'est plus là. Disparition, brutale, absolue; le regard de Scottie enregistre cette absence (comment figurer une absence? par un plan où ce qu'on sattend à voir n'y est pas $^{9}$ ). Mouvement tournant de ce plan-regard, qui petit à petit découvre $M$ adeleine, blanche et auratique, adossée à un arbre; elle était simplement cachée derrière des troncs. Scène limpide en termes de scénario: la supercherie Judy-M adeleineCarlotta est à son comble, Scottie est parfaitement aveugle parce que totalement ébloui. Scène à double fond, en termes figuratifs: I'enjeu est de séparer visuellement les deux personnages, de leur donner deux lieux qui ne communiquent pas. Elle échappe à son regard, à son espace; dans la conversation qui suit, ils sont assignés l'un et l'autre à une moitié du cadre, rigidement. D isparition, cloisonnement des espaces: c'est cela que la double scène d'hôtel doit reprendre, replier autrement, corriger, rectifier.

Apparition de lumière; disparition dans un espace inexistant, fait de pure absence; fausses annonces, bluff de la prophétie et de la voyance. Confrontation d'un annunziato d'occasion à des «messages» truqués. (H itchcock a-t-il pensé peinture? Sûrement pas, mais en pensant mise en scène il a laissé le cinéma classique y penser à sa place.)

Scène mystique, l'Annonciation a perdu peu à peu, dans la peinture, sa force d'émerveillement devant le miracle, parce que la connivence - de la spéculation théorique et de la mise en scène - s'est épuisée à force d'évidence. C'est pourquoi on est passé à un autre registre, celui du lumen/numen. $O r$, les nuages de lumière, les plongées ou contre-plongées (Tiepolo) vertigineuses, les espaces béants, sans murs, sans architecture et presque sans sol (Titien, à la fin de sa vie), tout cela n'était pas maté riau du miracle et du mystère - mais de plus en plus, matériau de peinture, éloigné de toute transcendance et très présent physiquement. Le miracle de l'art se sépare de plus en plus du miracle évangélique, à mesure que l'artiste saffirme comme maître d'œuvre. $D$ ans ce mouvement d'idées qui fait venir au premier plan la figure du peintre, la mise en scène joue un rôle essentiel : le grand peintre est celui qui se réapproprie les grandes scènes canoniques, celle de l'Annonciation et les autres, pour se définir comme auteur de peinture, quand au siècle précédent la 
variation, si inventive qu'elle fût, laissait toujours affleurer le même rapport intellectuel et spirituel au texte. La question est donc bien celle de la mise en scène, que la peinture découvre longtemps avant le thêâtre, et dans une histoire proche d'une certaine histoire du cinéma.

Proust et la Berma: toute la découverte du narrateur, c'est que l'art de la Berma n'est pas un supplément plus ou moins mobile, plus ou moins génial, ajouté à un rôle neutre qui serait le même pour toutes les actrices, quel que soit «par ailleurs» leur talent. L'art de la Berma, c'est le rôle lui-même, inséparable de la manière dont elle le joue. L'école des Cahiers a été proustienne sans le savoir, refusant toute conception de la mise en scène comme supplément détachable (que l'on pourrait par conséquent apprendre, voire dans des écoles), valorisant au contraire des cinéastes chez qui tout, mise en scène, choix des histoires et des personnages, tenue du récit, présence de l'auteur dans son œuvre, est indissociable, et relève d'un seul et même exercice de l'art. ( $D$ e même encore que $M$ anet se distingue $d^{\prime} I n$ gres par le choix de ses sujets autant que par leur mise en scène et par la touche picturale. $M$ ais n'est-ce pas là un problème éminemment moderne?)

D onc «faire Annonciation », cela n'a pas été un supplément détachable, une volonté de citer, de s'ennoblir ou de s'inscrire dans qui sait quel héritage. $\mathrm{Ni}$ Godard ni Pasolini font spécialement geste de peintre en reproduisant quelque chose du dispositif de l'Annonciation : ils ont fait geste d'auteurs. Ils ont démontré leur désir de faire du cinéma, c'est-à-dire de manier des formes, des thèmes, des figures, ensemble. Si Pasolini a pu rêver d'être Giotto, ou Godard, M anet, ce n'est qu'en tant que ces peintres étaient des auteurs, au sens de la «politique des auteurs», et que leur mise en scène était leur principal moyen d'expression.

On peut voir en cinéma des Annonciations partout (c'est la tendance de Bergala). Et pourquoi pas? «N ous n'avons pas d'autre culture» en effet ${ }^{10}$, et chez des cinéastes suffisamment vieux, le fonds chrétien est encore prégnant. Le bénéfice interprétatif, cependant, samincit à mesure qu'enfle la masse des occurrences. $\mathrm{Si}$ trop de scènes de films sont lisibles comme l'écho, même 
inversé, de la conversation de $\mathrm{M}$ arie et de l'Ange, cela ne signifiera bientôt plus rien, que la connivence interminée et indéterminée de nos images avec les mythes chrétiens, eux-mêmes privés de substance si on les ramène par trop à des schèmes, si l'on oublie leur savoureux, leur catholique détail (ce pourquoi j'ai cité $H$ itch, catholique jusqu'au bout des ongles dans son amour des minuties). O n peut donc aussi, et plus sobrement, prétendre qu'en réalité il n'y a nulle part de vraies Annonciations de cinéma - même chez Godard, qui a beaucoup rhabillé le théâtre évangélique. Pas de vraie Annonciation, là où la religion n'est plus qu'un souvenir de religion, un petit morceau de génome culturel, une équivoque permanente du croire-ne pas croire.

J'essaie d'échapper à la fausse radicalisation de ce «nulle part » et de ce «partout» pseudo-pascaliens, de ne pas parier sur la reprise, ou non, des schèmes chrétiens et picturaux. II y a rencontre dans l'attitude d'artiste; il y a connivence sur l'idée de mise en scène; il y a des problèmes particuliers qui se ressemblent (et il y a, très secondairement, une iconographie singulière). Ce qui se profile derrière la reprise du dispositif - catholique et dans son détail - de l'Annonciation, c'est la migration, c'est l'auteur, c'est l'art. (À tout prendre, à l'attitude qui consiste à voir le même contenu partout sous des formes différentes, je préfère l'attitude lévistraussienne - voir des contenus sans cesse changeants sous des formes identiques.)

L'H omme qui rétrécit: un nuage le contamine. The Fog (C arpenter) : le nuage prend consistance, humanoïde, comme le gros nuage gris et pataud qui figure Jupiter dans le tableau de Corrège à V ienne. LeVillage des damnés, les deux versions, surtout celle de Carpenter qui insiste sur le caractère rayonnant de l'apparition des enfants monstres. Body Snatchers de Ferrara et Kaufman. L'O mbre d'un doute (la fumée noire lorsque l'oncle Charlie arrive en ville). Rendez-vous avec la peur (Tourneur). Etc. Le cinéma a lui aussi son travail du lumineux; mais sur ce terrain il ne peut se confondre ni même se réunir tout à fait avec la peinture. Les effets de lumière ne sont intéressants au cinéma, ils n'ont la chance de faire une image que si ce sont des effets temporels (dans le mouvement ou dans le montage). $D$ ans les films de terreur, ce qui est vivant, ce ne sont pas les créatures plus ou moins 
artificielles qu'inventent les scénarios (encore que la fiction des accapareurs de corps soit l'une des plus profondes jamais imaginées, digne des grands scénarios romantiques du vol d'âme ou du vol d'ombre). C'est l'art des nuées et de leur existence dans le temps, et c'est pourquoi ils n'ont guère de ressemblance avec la peinture.

Autant de films, de genres, de cinéastes (C arpenter, Cronenberg, Lynch et d'autres) pour qui I'annonciation, le trait annonciateur d'un être ou d'un phénomène, n'a pas besoin de repasser par l'épreuve de la peinture, de l'histoire des images, de la connivence d'une spéculation et d'un point de dogme. Cinéma et peinture: la migration, d'objets, de symbolismes, de dispositifs et de mises en scène, n'est pas indispensable. Resterait, pour continuer, à se demander comment, où, pourquoi des images comme celles que permet un genre (en l'occurrence, «fantastique») sont aussi, d'une certaine façon, destinées à relever celles de la peinture. «N ous n'avons pas d'autre culture. »

\section{Université de Paris III et EH ESS}

\section{NOTES}

1. Cet article se rattache à un projet plus général d'étude des «migrations» formelles entre le cinéma et la peinture. D eux autres moments de la même réflexion d'ensemble ont déjà été publiés: «M igrations», Cinémathèque, n 7, 1995, p. 35-47, et «Vanités (M igrations, 2)», Cinémathèque, n 16, 1999, p. 7-21.

2. Presque en même temps parurent en France le livre de Pascal Bonitzer, $D$ écadrages (Paris, Cahiers du Cinéma/Éditions de l'Étoile, 1995), mon ouvrage L'CE il interminable (Paris, Librairie Séguier, 1995) et les actes du colloque de Chantilly, Cinéma et peinture, dirigé par Raymond Bellour (Paris, P.U.F., 1990).

3. Voir Aumont (1999).

4. Voir la description synthétique de ces attitudes traditionnelles dans M ichael Baxandall (1972).

5. Voir Bergala (1993), «M ontage obligatoire». Ce texte donnait d'autres exemples de reprise virtuelle du dispositif annonciatif, et a donc été l'un des moteurs de mes présentes remarques. Bergala a repris et développé la partie de son texte consacrée à Je vous salue M arie dans son ouvrage N ul mieux que Godard (1999).

6. Cf. Baxandall (1972).

7. J'ai évoqué une autre manière de rattacher à l'héritage chrétien ce «personnage» joué par Silvia Pinal, dans «Et D ieu... créa la femme» (Aumont, 2002).

8. O n sait que le sous-texte du Couvent est très largement constitué d'une allusion élaborée aux deux Faust de G oethe, mais dans cette scène en particulier, cela n'empê- 
che pas l'utilisation, pour la mise en scène de ce qui est une apparition semiprophétique, du dispositif «annonciatif».

9. Voir Emmanuel Siety (1998).

10. La formule est due à J ean Louis Schefer $(1998, p .15)$ : «Q uoi que nous fassions, pensions, nous n'avons pas d'autre histoire ni d'autre culture. »

\section{RÉFÉREN CES BIBLIOGRAPHIQUES}

Arasse, 1999: D aniel Arasse, L'Annonciation italienne, Paris, H azan, 1999.

Aumont, 1999: Jacques Aumont, «Vanités (M igrations, 2) », Cinémathèque, n 16 , 1999, p. 7-21.

Aumont, 2002 : Jacques Aumont, «Et Dieu... créa la femme», dans J.-Cl. Schmitt (dir.), Ėveet Pandora. La création dela première femme, Paris, Gallimard, 2002.

Baxandall, 1972: M ichael Baxandall, Painting and Experience in Fifteenth Century Italy, O xford, O xford University Press, 1972.

Bergala, 1993: Alain Bergala, «M ontage obligatoire», dans Jacques Aumont (dir.), Le M ontage dans tous ses états, conférences du C.H .A.C., Cinémathèque française, 1993.

Bergala, 1999 : Alain Bergala, Nul mieux que Godard, Paris, Cahiers du cinéma, 1999.

Schefer, 1998: Jean Louis Schefer, Cinématographies. O bjets périphériques et mouve ments annexes, Paris, P.O .L., 1998.

Siety, 1998 : Emmanuel Siety, «Ce qui manque et ce qui reste: disparition, abandon, effacement », Cinémathèque, nº 14, 1998, p. 85-98. 